\title{
Brain motor control assessment post intensive whole-body exercise vs. upper body exercise after spinal cord injury
}

\author{
Maryam Zoghi' ${ }^{1}$ Mary Galea ${ }^{2}$ \\ 'Department of Physiotherapy, Podiatry, and Prosthetics and Orthotics, LaTrobe University, Bundoora, Victoria 3086, Australia. \\ ${ }^{2}$ Department of Medicine, The University of Melbourne, Parkville, Victoria 3010, Australia.
}

Correspondence to: Dr. Maryam Zoghi, Department of Physiotherapy, Podiatry, and Prosthetics and Orthotics, LaTrobe University, Bundoora, Victoria 3086, Australia. E-mail: m.zoghi@latrobe.edu.au

How to cite this article: Zoghi M, Galea M. Brain motor control assessment post intensive whole-body exercise vs. upper body exercise after spinal cord injury. Neuroimmuno/ Neuroinflammation 2019;6:14. http://dx.doi.org/10.20517/2347-8659.2019.03

Received: 8 Jul 2019 First Decision: 23 Sep 2019 Revised: 24 Nov 2019 Accepted: 5 Dec 2019 Published: 17 Dec 2019

Science Editor: Swapan K. Ray Copy Editor: Jing-Wen Zhang Production Editor: Tian Zhang

\begin{abstract}
Aim: The aim of this study was to assess the pattern of voluntary movements in patients with spinal cord injury (SCI) post intensive whole-body training $v s$. upper body training with brain motor control assessment (BMCA).

Methods: Twelve neurologically intact participants and 18 patients with $\mathrm{SCl}$ participated in this study as part of a multi-centre randomised controlled trial. All participants received 12 weeks training (three times per week), which comprised trunk, upper and lower limb exercises and locomotor training and functional electrical stimulation-assisted cycling in whole-body training group and an upper body strength and fitness program for upper body training group.
\end{abstract}

Results: Generalised linear model analysis showed significant effect of the main effect of the Task $(P<0.001)$ on the similarity index of voluntary movement patterns but not on the other factors or the interactions between them $(P>0.05)$. Some participants showed significant improvement in muscle strength post 12 weeks training; however, this improvement was not reflected in the pattern of muscle activation which was captured by BMCA.

Conclusion: BMCA is a valuable objective assessment tool that could add resolution to the clinical evaluation of patients with $\mathrm{SCI}$ post different therapeutic techniques.

Keywords: Brain motor control assessment, spinal cord injury, discomplete

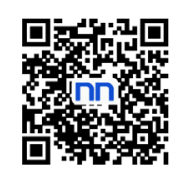




\section{INTRODUCTION}

Spinal cord injury (SCI) is one of the most devastating disabilities which can affect a person's life significantly. Normal movement patterns are significantly impaired as a result of a spinal lesion, due to decreased/loss of supraspinal influences over the spinal cord and impaired appreciation of peripheral sensory inputs. One of the main aims of rehabilitation for patients with SCI is to assist them to become as independent as possible in daily activities ${ }^{[1]}$ and facilitate normal movement patterns as much as possible. For patients with complete SCI, however, therapists usually do not focus on promoting neurological improvement in the paralysed extremities. For this group of patients, rehabilitation strategies are mainly focused on teaching compensatory strategies including using a variety of assistive devices during therapeutic sessions ${ }^{[1]}$.

Many patients considered to have clinically complete SCI are neurophysiologically incomplete (discomplete $)^{[2-6]}$. It has been argued that these connections are not detectable with clinical assessment; however, they are able to modulate the excitability of spinal sensorimotor connections below the level of injury $^{[6-8]}$. In addition, there has been a case where the function of muscles below the level of injury could be improved by using functional electrical stimulation (FES) or locomotor training (LT) while body weight is partially supported ${ }^{[9-1]}$.

Brain motor control assessment (BMCA) is a surface electromyography-based assessment that can add resolution to clinical assessment in patients with $\mathrm{SCI}^{[12]}$. In this assessment, motor outputs from the nervous system are recorded through a variety of reflexes and voluntary motor tasks of the lower limbs ${ }^{[13]}$ performed under strictly controlled conditions. Sub-clinical evidence of translesional motor connections has been observed in patients considered to have a clinically complete lesion of the spinal cord using this type of assessment ${ }^{[2]}$. These subclinical responses can take various forms, for example repeatable responses to reinforcement manoeuvres or strong vibration ${ }^{[14]}$ or the ability to volitionally suppress responses evoked by plantar surface stimulation ${ }^{[15,16]}$.

This paper presents the results of the BMCA assessments conducted in patients at one site of a multi-centre, assessor-blinded, randomised controlled trial (Spinal Cord Injury and Physical Activity Full-On) ${ }^{[17]}$, which investigated the effectiveness of an intensive activity-based therapy program for patients with clinically complete and incomplete SCI. For full details of the protocol, please refer to Galea et al. ${ }^{[17]}$. The trial was registered on ClinicalTrials.gov (NCT01236976).

\section{METHODS}

Twelve neurologically intact participants (six female and six male) and 18 patients who were at least 6 months post-SCI participated in this study. The demographic information of patients with SCI including neurological level (sensory and motor) and American Spinal Injury Association Impairment Scale (AIS) classification are presented in Table $1^{[18]}$. These measurements were generated using the International Standard for Neurological Classification of Spinal Cord Injury (ISNCSCI) ${ }^{[18,19]}$.

All participants gave their written informed consent before the BMCA assessments were carried out (in addition to the consent for the clinical trial). All procedures used conformed with the Declaration of Helsinki, and the protocol was approved by the Human Research Ethics Committees at The University of Melbourne and Austin Health.

Neurologically intact participants were assessed using BMCA to generate prototype response vectors for two bilateral voluntary tasks (hip/knee flexion-extension) and four unilateral voluntary tasks (hip/knee flexion/extension and ankle dorsiflexion/plantar flexion). These values were used to calculate the similarity 
Table 1. Demographics of patients with spinal cord injury

\begin{tabular}{|c|c|c|c|c|c|}
\hline \multirow{2}{*}{ Participant/Ax } & \multicolumn{4}{|c|}{ Neurological level } & \multirow{2}{*}{ AIS } \\
\hline & Sensory right & Sensory left & Motor right & Motor left & \\
\hline P1 & T6 & T6 & T6 & T6 & $\mathrm{D}$ \\
\hline P2 & $\mathrm{C} 8$ & $\mathrm{C7}$ & $\mathrm{T} 1$ & $\mathrm{~T} 1$ & B \\
\hline P3 & $\mathrm{C} 1$ & $\mathrm{C} 3$ & C6 & $\mathrm{C} 3$ & D \\
\hline P4 & T6 & $\mathrm{T7}$ & $\mathrm{T} 1$ & $\mathrm{~T} 1$ & $A$ \\
\hline P5 & $\mathrm{C} 7$ & $\mathrm{~T} 12$ & $\mathrm{~T} 1$ & $\mathrm{~T} 12$ & $\mathrm{D}$ \\
\hline P6 & $\mathrm{C} 2$ & $\mathrm{C} 2$ & $\mathrm{C} 2$ & $\mathrm{C} 2$ & $\mathrm{D}$ \\
\hline P7 & $\mathrm{C} 5$ & $\mathrm{C7}$ & $\mathrm{C} 5$ & S1 & $\mathrm{D}$ \\
\hline P8 & $\mathrm{T} 2$ & T3 & $\mathrm{T} 2$ & T3 & $A$ \\
\hline P9 & $\mathrm{C} 5$ & $\mathrm{C} 5$ & C6 & C6 & $A$ \\
\hline P10 & T8 & T8 & T8 & T8 & A \\
\hline P11 & $\mathrm{C} 3$ & $\mathrm{C} 3$ & $\mathrm{C} 6$ & C6 & A \\
\hline P12 & T5 & T6 & T5 & T6 & $A$ \\
\hline P13 & $\mathrm{T} 4$ & T5 & $\mathrm{T} 4$ & T5 & $A$ \\
\hline P14 & $\mathrm{C} 8$ & $\mathrm{C} 8$ & $\mathrm{~T} 1$ & $\mathrm{~T} 1$ & B \\
\hline P15 & $\mathrm{T} 1$ & $\mathrm{~T} 2$ & T1 & T2 & C \\
\hline P16 & C8 & C8 & T1 & $\mathrm{T} 1$ & A \\
\hline P17 & T4 & T4 & T4 & T4 & A \\
\hline P18 & T3 & T3 & T3 & $\mathrm{T} 1$ & A \\
\hline
\end{tabular}

T: thoracic; C: cervical; S: sacral; Ax: assessment; AIS: association impairment scale; P: participant

index (SI) value for each task ${ }^{[20,21]}$. The relative distribution of surface EMG (sEMG) activity across the chosen muscles for each lower limb task (presented according to SI) in patients with SCI were compared to SI values for each task in neurologically intact participants.

Inclusion criteria for patients with $\mathrm{SCI}^{[22]}$ were: $\geq 18$ years old and able to give informed consent; sustained a traumatic SCI $\geq 6$ months prior to consent and had completed their primary rehabilitation; and had a complete or incomplete SCI $(\mathrm{C} 6-\mathrm{T} 12)^{[17]}$. Exclusion criteria for patients with SCI ${ }^{[22]}$ were: brachial plexus, cauda equina or peripheral nerve injury; Stage 3 or 4 pressure ulcer ${ }^{[23]}$; had recent major trauma or surgery (up to six months prior to this trial); were post-menopausal at the time of injury (females); had a BMI < 25; had endocrinopathy or metabolic disorders of the bone; had a medical history of exposure to medication(s) known to affect mineral or bone metabolism; had chronic systemic diseases; had significant impairment or disability; had severe spasticity; had uncontrolled neuropathic pain; were likely to experience clinically significant autonomic dysreflexia and/or orthostatic hypotension in response to electrical stimulation or prolonged upright postures; or had any contraindications to FES such as a cardiac pacemaker, lower limb fracture or pregnancy.

Patients with SCI were randomly allocated to whole body exercise or upper body exercise groups [Table 2]. Twenty-four potential participants were screened for this trial at this centre. One failed to meet the inclusion criteria. Three were withdrawn after the first assessment based on personal reasons and two were not available for the BMCA assessments. Therefore, data from 18 patients with SCI were included in data analysis. Participants in the whole-body exercise group ( $n=12$; 7 AIS A complete; 5 AIS B-D incomplete) participated in training sessions three times per week for 12 weeks, which comprised trunk, upper and lower limb exercises, LT and FES-assisted cycling. LT sessions ${ }^{[22]}$ were provided using a Therastride system (Innoventor, Inc., St Louis, MO, USA). Participants were supported in a harness. One therapist stood behind the participant to assist them to maintain optimal posture and facilitate rotation of the pelvis, while two therapists/assistants moved the lower limbs during the training session. The treadmill speed was adjusted individually based on the stepping pattern and body weight load. It was progressively increased as appropriate to a normal walking speed range $(0.89-1.34 \mathrm{~m} / \mathrm{s})$. In addition, as participants were improving, the amount of body weight support was gradually reduced ${ }^{[24]}$. FES-assisted cycling was provided using a 
Table 2. Group allocation of each participant and time of each assessment post injury

\begin{tabular}{|c|c|c|c|c|c|c|c|}
\hline Participant & Group WBT or UBT & Date of injury & Level of injury & First Ax WPI & Second Ax WPI & Third Ax WPI & Fourth Ax WPI \\
\hline P1 & WBT & $06 / 2009$ & D Incomplete & 91 & 107 & 120 & 147 \\
\hline P2 & WBT & $03 / 2009$ & B Incomplete & 152 & 167 & NA & NA \\
\hline P3 & WBT & $04 / 2002$ & D Incomplete & 527 & 543 & 553 & NA \\
\hline P4 & WBT & $07 / 2010$ & A Complete & 83 & 113 & 124 & NA \\
\hline P5 & UBT & $10 / 2009$ & D Incomplete & 93 & 108 & 122 & 148 \\
\hline P6 & WBT & $12 / 1960$ & D Incomplete & 2641 & 2657 & 2673 & 2697 \\
\hline P7 & UBT & $08 / 2010$ & D Incomplete & 91 & 107 & 118 & NA \\
\hline P8 & WBT & $06 / 2006$ & A Complete & 243 & 262 & 273 & 300 \\
\hline P9 & WBT & $05 / 2001$ & A Complete & 592 & 605 & NA & NA \\
\hline P10 & UBT & $11 / 2002$ & A Complete & 511 & 525 & NA & NA \\
\hline P11 & WBT & $04 / 2006$ & A Complete & 320 & 337 & 346 & NA \\
\hline P12 & UBT & $12 / 2008$ & A Complete & 115 & 133 & 146 & 172 \\
\hline P13 & UBT & $08 / 2010$ & A Complete & 109 & 122 & NA & NA \\
\hline P14 & UBT & $06 / 2011$ & B Incomplete & 6 & 22 & 41 & 61 \\
\hline P15 & WBT & 03/1999 & C Incomplete & 647 & 663 & 678 & 703 \\
\hline P16 & WBT & $10 / 2001$ & A Complete & 554 & 570 & 580 & NA \\
\hline P17 & WBT & $08 / 2003$ & A Complete & 418 & 433 & NA & NA \\
\hline P18 & WBT & 09/1992/ & A Complete & 961 & 979 & 991 & 1019 \\
\hline
\end{tabular}

WBT: whole body training; UBT: upper body training; WPI: weeks post injury; NA: not assessed; Ax: assessment; P: participant

RT300 cycle (Restorative Therapies, Baltimore, MD, USA). Surface electrodes were attached on quadriceps, gluteal and hamstrings muscles. The parameters of the FES were: pedal cadence, 5-50 rev/min; stimulus intensity, maximum $140 \mathrm{~mA}$; pulse width, $0.3 \mathrm{~ms}$; frequency, $35 \mathrm{~Hz}$; and duration, up to $30 \mathrm{~min}^{[24]}$.

Participants in the upper body exercise group ( $n=6,3$ AIS A complete and 3 AIS B-D incomplete) received an upper body strength and fitness program three times per week for 12 weeks. This upper body training program included a circuit-based exercise program incorporating resistance and cardiorespiratory training. None of the participants had participated in an intensive exercise program during the three-week period before starting this trial.

The participants with SCI were assessed up to four times over a period of one year. The assessment sessions are reported based on the number of weeks post-SCI [Table 2].

The following assessments were performed on participants in both groups before training (baseline), after 12 weeks of training and 6 months and 12 months post-recruitment.

\section{Lower limb BMCA}

The lower limb BMCA protocol was performed with participants lying supine. The protocol included: voluntary tasks, tendon-tap responses and vibration responses. The sEMG of 14 muscles (seven muscles from each lower limb and trunk) were recorded continuously throughout the protocol ${ }^{[21]}$ with self-adhesive pre-gelled disposable surface electrodes (Noraxon Dual electrodes, Scottsdale AZ, USA). The muscles were lumbar paraspinal muscles, rectus abdominis, quadriceps, adductors, tibialis anterior, hamstring and gastrocnemius. EMG signals were amplified $(1000 \times)$ by ZeroWire electrodes (Cometa, Milan, Italy) and then filtered $(20-500 \mathrm{~Hz})$ and digitised online $(1 \mathrm{kHz}$ sampling rate) using a PowerLab recording system (ADInstruments Ltd).

Two bilateral voluntary tasks (hip/knee flexion-extension) and four unilateral voluntary tasks (hip/knee flexion/extension and ankle dorsiflexion/plantar flexion) were assessed on both sides. All voluntary tasks were cued by two 5 -s tones with a brief pause (less than $1 \mathrm{~s}$ ) between them. Participants were asked to start the first task at the tone and not to start the second task until they heard the second tone. A customised 
tendon hammer was used to record ten tendon responses quadriceps and triceps surae bilaterally with similar strike (consistent energy and independent of orientation and relative position) ${ }^{[21]}$. Tonic vibratory responses (TVR) of quadriceps and triceps surae muscles on both sides were also assessed by applying 30-s vibration over the tendon. The vibrator was custom-constructed from a pneumatic hand-grinder fitted with an offset weight and protective barrel (frequency of $115 \mathrm{~Hz}$ and a motion amplitude of $0.8 \mathrm{~mm}$ peak to peak). All assessments were completed by an assessor blinded to group allocation.

\section{ISNCSCI}

The motor scores were derived from part of the ISNCSCI assessment ${ }^{[19]}$. It involved testing the strength of ten key muscles on each side of the body in the supine position (elbow flexors, wrist extensors, elbow extensors, finger flexors, finger abductors, hip flexors, knee extensors, ankle dorsiflexors, long toe extensors and ankle plantar flexors) on a scale from $0=$ no contraction to $5=$ normal resistance through full range of motion. Scores were summed to give a total possible score of 50 for the upper extremities and 50 for the lower extremities.

\section{Data reduction}

A prototype response vector for each phase of each voluntary task in the protocol was generated from 12 neurologically intact participants $(24 \mathrm{limbs})^{[20,21]}$. The muscles selected for hip and knee tasks in prototype calculations were quadriceps, hip adductors, hamstrings, lumbar paraspinal muscles and rectus abdominis from both sides. Those selected for ankle tasks in prototype calculations were quadriceps, hamstrings, tibialis anterior and gastrocnemius from both sides.

These values were used to calculate the SI, which compares the relative distribution of sEMG activity across the above chosen muscles for each voluntary tasks ${ }^{[21]}$ and to evaluate the progression of participants with SCI during the trial. If SCI participants were able to recruit the prime movers for a specific task and decrease unnecessary muscle activity in the other muscles, their SI scores approximated neurologically intact participants' values, indicating better control of their movements. A value of 1.0 for the SI means that the test participant had an identical distribution of sEMG activity across muscles to the neurologically intact group for that task.

Generalised linear model (GLM) analysis was used to assess the main effects of Group: whole body training vs. upper body training; Side: right $v s$. left; Tasks: four unilateral tasks on both sides; and Assessment timepoint (Ax): first Ax (baseline), second Ax (after 12 weeks training), third Ax (6 months postrandomisation) and fourth $A x$ (12 months post-randomisation), on SI. GLM analysis was also conducted to assess the main effects of Group (whole body training vs. upper body training) and Ax [first Ax (baseline), second Ax (after 12 weeks training), third Ax (6 months post-randomisation) and fourth Ax (12 months post-randomisation)] on ISNCSCI motor score for right upper and lower limbs, left upper and lower limbs and total ISNCSCI motor score for upper and lower limbs. A significance level of $P<0.05$ was adopted for all comparisons. This analysis was conducted using SPSS 22 software.

\section{RESULTS}

The main effect of Group, Side or Assessment time point on SI was not significant. GLM analysis only showed a significant main effect of Task $(P<0.001)$ on SI. There were no significant interactions between the factors $(P>0.05)$. The individual SI changes over time for two tasks in both groups are shown in Figure 1.

GLM analysis showed a significant main effect of Group $(P<0.05)$ on ISNCSCI motor scores of right, left and total ISNCSCI motor score for the upper limb [Figure 2]. However, the main effect of Assessment time point, the interaction between Group and Assessment time point and the analyses of ISNCSCI motor scores from lower limb were not significant $(P>0.05)$. The strength of the key lower limb muscles in 12 


\section{Upper body training group}
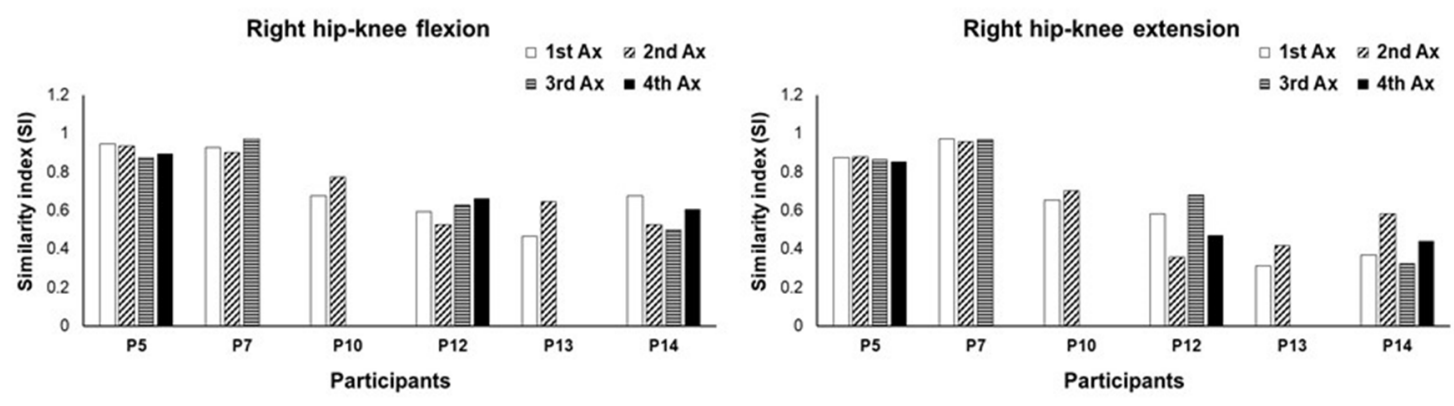

Whole body training group
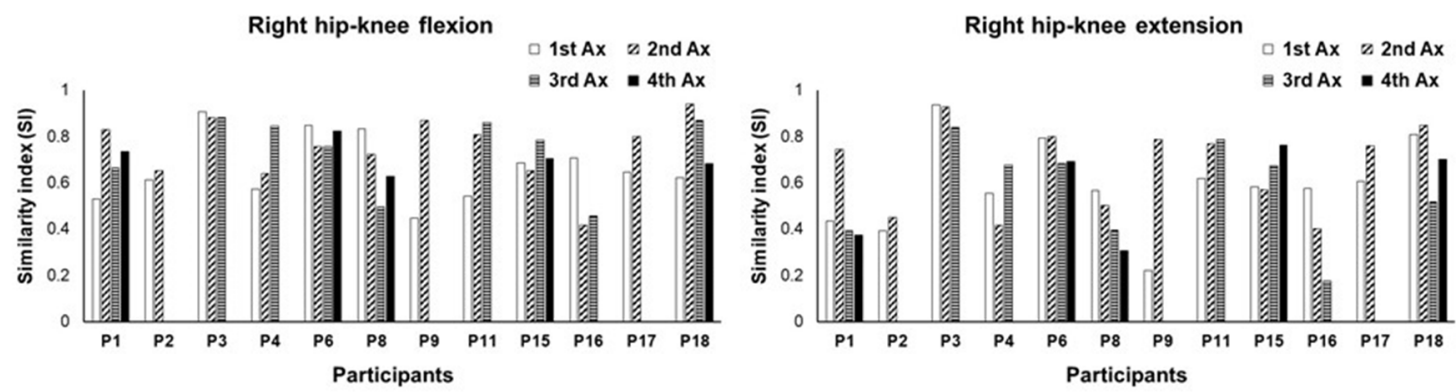

Figure 1. Individual SI changes over the 12 month-period and four assessment sessions for two tasks in both groups. Please note that some participants did not attend all the assessment sessions. Different participants showed different SI values for different tasks over time. P: participant; Ax: assessment; SI: similarity index

participants was documented as 0 at all assessment time points. Participants 1, 3, 5, 6, 7 and 15 showed some changes in lower limb muscle strength throughout the trial. Among these participants, Participant 5, who was in the upper body training group, showed a decrease in ISNCSCI motor scores; however, the other participants who showed no changes (Participant 7) or some improvements (Participants 3 and 6) in ISNCSCI motor scores were in the whole body training group.

Figure 3 shows the pattern of muscle activation during right and left hip-knee flexion and extension in a neurologically intact participant [Figure 3A] and two SCI participants (Participants 6 and 3) [Figure 3B and C] at four different assessment sessions throughout the trial in addition to the lower limb muscle strength changes during the trial for these two SCI participants [Figure $3 \mathrm{C}$ ]. Figure $3 \mathrm{C}$ shows the total of manual muscle testing scores for five key muscles (hip flexors, knee extensors, ankle dorsiflexors, long toe extensors and ankle plantar flexors) on right and left sides plus the total score for both sides for Participants 6 and 3 at four different assessment time points.

Figure $3 \mathrm{~A}$ shows the pattern of muscle activation during right and left hip-knee flexion and extension in a neurologically intact participant. Figure $3 \mathrm{~B}$ shows the pattern of muscle activation during the same tasks in Participant 6 (one of the participants with SCI) at four different assessment sessions throughout the trial. Figure $3 \mathrm{C}$ shows similar data as Figure $3 \mathrm{~B}$ in Participant 3 (another participant with SCI). Figure $3 \mathrm{C}$ shows the total of manual muscle testing scores for five muscles (hip flexors, knee extensors, ankle dorsiflexors, long toe extensors and ankle plantar flexors) on right and left side plus the total score for both sides for Participants 3 and 6 at four different assessment sessions. As can be seen in this figure, both Participants 3 and 6 showed some improvements in lower limb muscle strength throughout the trial at each assessment session; however, the increased strength of these muscles did not have any effect on the pattern of muscle activation during the assessed tasks (right and left hip-knee flexion and extension). For instance, the 


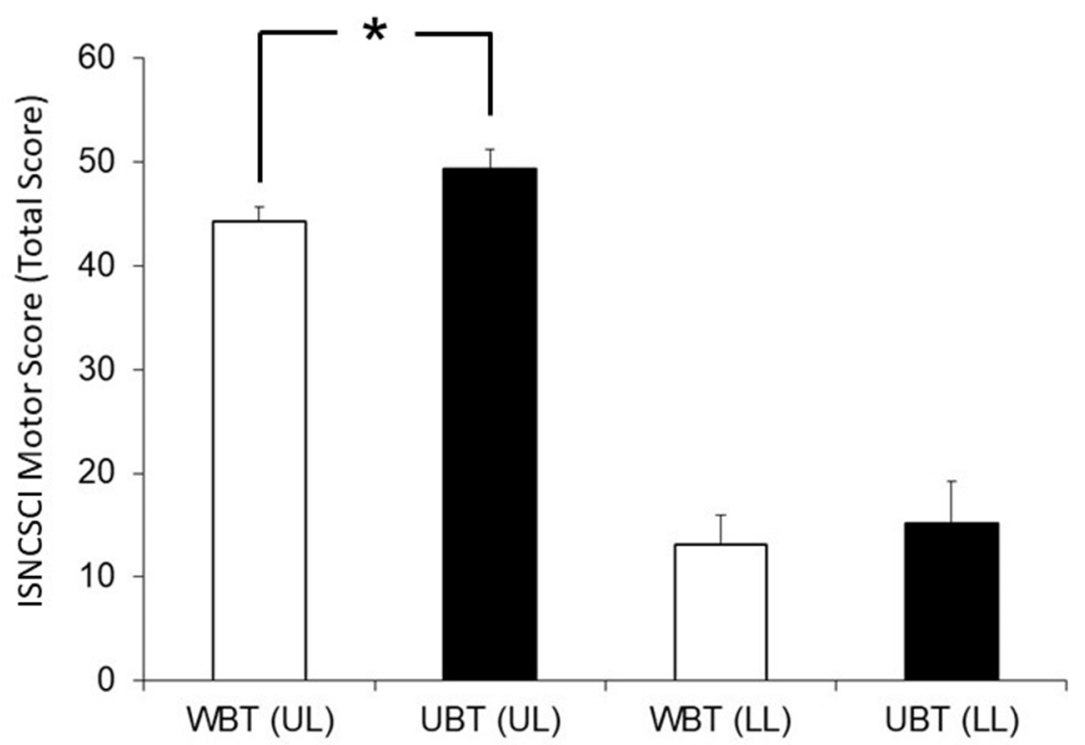

Figure 2. Total ISNCSCI motor score for upper and lower limbs in experimental and control group. WBT: whole-body training; UBT: upper body training; LL: lower limb; UL: upper limb; ISNCSCI: international standard for neurological classification of spinal cord injury

patterns of muscle activation during left hip-knee flexion in Participant 6 throughout the trial are very similar to each other and very different from the normal pattern of muscle activation [Figure 3A]. It can be seen that left quadriceps is more active than left hamstring and right hamstring does not show enough activity. The other example is during right hip-knee flexion: Participant 3 showed significant co-contraction of right hip adductors and left quadriceps even though they needed to be quiet during this task [Figure $3 \mathrm{~A}$ ].

In this study, 10 participants were assessed as having clinically complete SCI. Of these, nine participants showed tendon tap responses in 1-4 of the assessed muscles (Participants 4, 8, 10, 11, 12, 13, 16, 17 and 18) [Table 3]. Participants 4 and 11 did not show any TVR in any of the four assessed muscles. However, Participants 8, 10,12,13,16,17 and 18 showed TVR in 1-4 targeted muscles [Table 4].

Tendon tap responses are markers that can be used to indicate the existence of supraspinal influences over the motor circuitry of the examined muscle. Multi-level tendon-tap responses can be seen in some patients in both groups over time.

Vibration responses are markers that can be used to indicate the existence of supraspinal influences over the motor circuitry of the examined muscle. These responses were seen in some patients who were categorised as clinically complete SCI.

\section{DISCUSSION}

Eighteen participants with different levels of SCI (C6-T12) from one site who were participating in a multicentre randomised controlled trial were assessed up to four times with the BMCA protocol. Twelve of these participants received whole body training while the other six participants received an upper body strength and fitness program three times per week for 12 weeks. Five of the six participants in upper body training group had the maximum total ISNCSCI motor scores of 50 throughout the study as their injury levels were at the thoracic level or at $\mathrm{C} 8$ level (incomplete). The training provided to the whole-body group had no effect on lower limb ISNCSCI motor scores. Twelve of the 18 participants in this group were classified as AIS A-complete (10 participants) or AIS B (2 participants) with the strength of the assessed lower limb muscles recorded as 0 throughout the trial. 
A. Neurologically intact participant
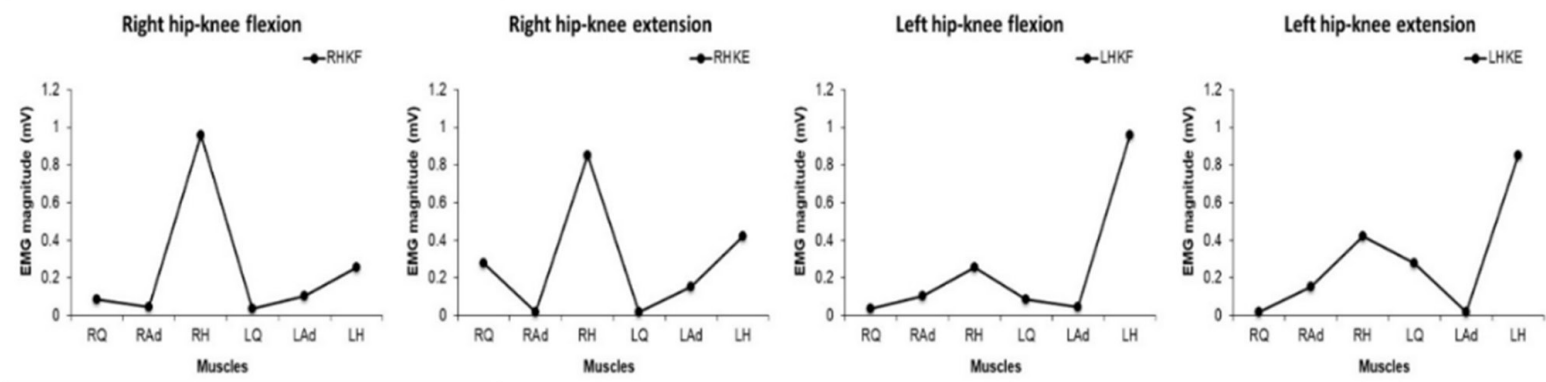

B. Participant 6
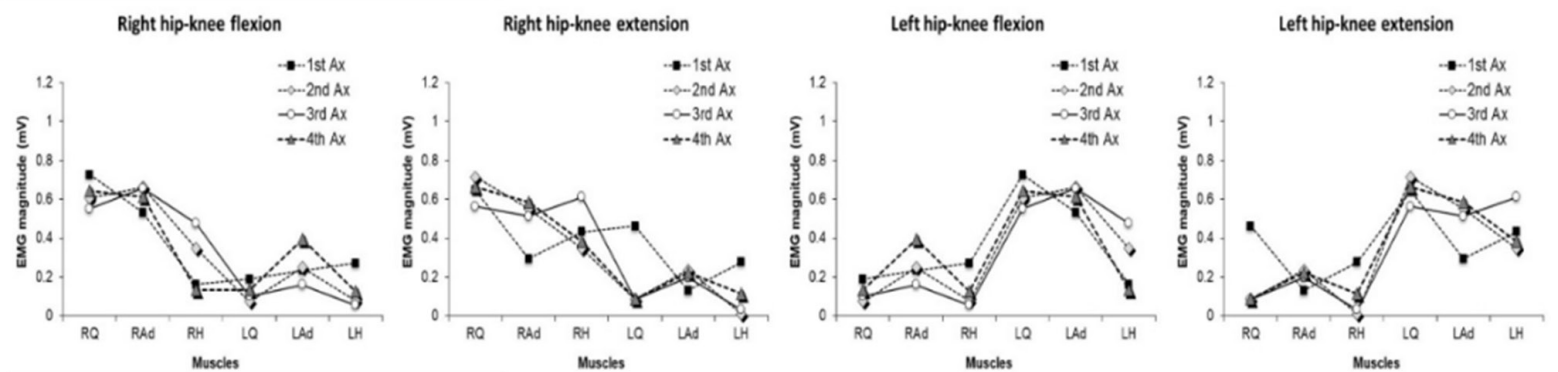

C. Participant 3
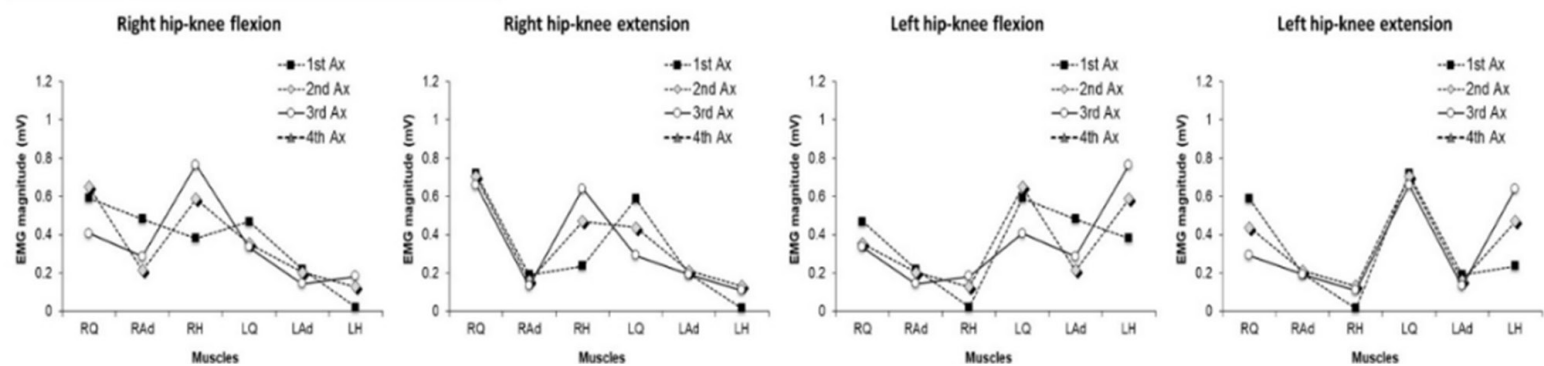

Figure 3. Pattern of muscle activation during right and left hip-knee flexion and extension in a neurologically intact participant (A) and $2 \mathrm{SCl}$ participants (B and $\mathrm{C}$ ). The lower limb muscle strength changes during the trial for these two $\mathrm{SCl}$ participants (C). RQ: right quadriceps; RAd: right hip adductors; RH: right hamstring; LQ: left quadriceps; LAd: left hip adductors; LH: left hamstring; Ax: assessment; $\mathrm{SCl}$ : spinal cord injury

BMCA can provide objective information regarding the pattern of muscular activation during lower limb tasks in patients with SCI during rehabilitation and how the treatment strategies can shift this pattern towards the normal pattern of movements in lower limbs. This assessment can be even more valuable when other functional clinical assessments, e.g., gait assessment (10-m walk test, timed up and go, 6-min walk test, etc.), cannot be completed due to the level of injuries, e.g., for patients with complete lesion at cervical or thoracic levels. Available data post BMCA are very limited, which makes it very difficult to compare these data with those of previous studies.

As shown in Figure 3, both Participants 3 and 6 (both in experimental group, incomplete D) showed some improvements in lower limb muscle strength throughout the trial at each assessment sessions; however, the increased strength of these muscles did not have any effect on the pattern of muscle activation during the assessed tasks (right and left hip-knee flexion and extension). For instance, the patterns of muscle activation during left hip-knee flexion in Participant 6 throughout the trial are very similar to each other and very different from the normal pattern of muscle activation [Figure $3 \mathrm{~A}]$. It can be seen that the left quadriceps are more active than the left hamstrings, and the right hamstrings do not show sufficient activity. Furthermore, during right hip-knee flexion, Participant 3 showed significant co-contraction of the right hip adductors and left quadriceps even though these muscles should have been quiet during this task 
Table 3. Tendon tap responses from right and left quadriceps and triceps surae during four assessment sessions in 18 participants with $\mathrm{SCl}$

\begin{tabular}{|c|c|c|c|c|}
\hline Participant/Complete vs. Incomplete & First Ax & Second Ax & Third Ax & Fourth Ax \\
\hline 1/Incomplete & $\begin{array}{l}\text { RQ: MLR } \\
\text { RTS: MLR } \\
\text { LQ: MLR } \\
\text { LTS: MLR }\end{array}$ & $\begin{array}{l}\text { RQ: MLR } \\
\text { RTS: MLR } \\
\text { LQ: MLR } \\
\text { LTS: MLR }\end{array}$ & $\begin{array}{l}\text { RQ: MLR } \\
\text { RTS: MLR } \\
\text { LQ: MLR } \\
\text { LTS: MLR }\end{array}$ & $\begin{array}{l}\text { RQ: MLR } \\
\text { RTS: MLR } \\
\text { LQ: MLR } \\
\text { LTS: MLR }\end{array}$ \\
\hline 2/Incomplete & $\begin{array}{l}\text { RQ: RP } \\
\text { RTS: RP } \\
\text { LQ: RP } \\
\text { LTS: RP }\end{array}$ & $\begin{array}{l}\text { RQ: MLR } \\
\text { RTS: MLR } \\
\text { LQ: MLR } \\
\text { LTS: MLR }\end{array}$ & NA & NA \\
\hline 3/Incomplete & $\begin{array}{l}\text { RQ: MLR } \\
\text { RTS: RP } \\
\text { LQ: MLR } \\
\text { LTS: RP }\end{array}$ & $\begin{array}{l}\text { RQ: MLR } \\
\text { RTS: RP } \\
\text { LQ: MLR } \\
\text { LTS: RP }\end{array}$ & $\begin{array}{l}\text { RQ: MLR } \\
\text { RTS: RP } \\
\text { LQ: MLR } \\
\text { LTS: RP }\end{array}$ & NA \\
\hline 4/Complete & $\begin{array}{l}\text { RQ: RP } \\
\text { RTS: NR } \\
\text { LQ: NR } \\
\text { LTS: RP }\end{array}$ & $\begin{array}{l}\text { RQ: RP } \\
\text { RTS: NR } \\
\text { LQ: NR } \\
\text { LTS: RP }\end{array}$ & $\begin{array}{l}\text { RQ: NR } \\
\text { RTS: NR } \\
\text { LQ: NR } \\
\text { LTS: RP }\end{array}$ & NA \\
\hline 5/Incomplete & $\begin{array}{l}\text { RQ: NR } \\
\text { RTS: NR } \\
\text { LQ: NR } \\
\text { LTS: NR }\end{array}$ & $\begin{array}{l}\text { RQ: MLR } \\
\text { RTS: NR } \\
\text { LQ: MLR } \\
\text { LTS: NR }\end{array}$ & $\begin{array}{l}\text { RQ: NR } \\
\text { RTS: NR } \\
\text { LQ: NR } \\
\text { LTS: NR }\end{array}$ & $\begin{array}{l}\text { RQ: MLR } \\
\text { RTS: NR } \\
\text { LQ: MLR } \\
\text { LTS: NR }\end{array}$ \\
\hline $6 /$ Incomplete & $\begin{array}{l}\text { RQ: MLR } \\
\text { RTS: NR } \\
\text { LQ: MLR } \\
\text { LTS: NR }\end{array}$ & $\begin{array}{l}\text { RQ: MLR } \\
\text { RTS: NR } \\
\text { LQ: MLR } \\
\text { LTS: RP }\end{array}$ & $\begin{array}{l}\text { RQ: MLR } \\
\text { RTS: NR } \\
\text { LQ: RP } \\
\text { LTS: NR }\end{array}$ & $\begin{array}{l}\text { RQ: MLR } \\
\text { RTS: NR } \\
\text { LQ: MLR } \\
\text { LTS: NR }\end{array}$ \\
\hline 7/Incomplete & $\begin{array}{l}\text { RQ: MLR } \\
\text { RTS: RP } \\
\text { LQ: NR } \\
\text { LTS: RP }\end{array}$ & $\begin{array}{l}\text { RQ: MLR } \\
\text { RTS: RP } \\
\text { LQ: MLR } \\
\text { LTS: RP }\end{array}$ & $\begin{array}{l}\text { RQ: MLR } \\
\text { RTS: RP } \\
\text { LQ: MLR } \\
\text { LTS: RP }\end{array}$ & NA \\
\hline 8/Complete & $\begin{array}{l}\text { RQ: MLR } \\
\text { RTS: RP } \\
\text { LQ: NR } \\
\text { LTS: RP }\end{array}$ & $\begin{array}{l}\text { RQ: MLR } \\
\text { RTS: RP } \\
\text { LQ: MLR } \\
\text { LTS: RP }\end{array}$ & $\begin{array}{l}\text { RQ: NR } \\
\text { RTS: RP } \\
\text { LQ: NR } \\
\text { LTS: NR }\end{array}$ & $\begin{array}{l}\text { RQ: NR } \\
\text { RTS: RP } \\
\text { LQ: NR } \\
\text { LTS: NR }\end{array}$ \\
\hline 9/Complete & $\begin{array}{l}\text { RQ: MLR } \\
\text { RTS: MLR } \\
\text { LQ: MLR } \\
\text { LTS: MLR }\end{array}$ & $\begin{array}{l}\text { RQ: MLR } \\
\text { RTS: MLR } \\
\text { LQ: MLR } \\
\text { LTS: MLR }\end{array}$ & NA & NA \\
\hline 10/Complete & $\begin{array}{l}\text { RQ: RP } \\
\text { RTS: MLR } \\
\text { LQ: MLR } \\
\text { LTS: MLR }\end{array}$ & $\begin{array}{l}\text { RQ: NR } \\
\text { RTS: MLR } \\
\text { LQ: MLR } \\
\text { LTS: MLR }\end{array}$ & NA & NA \\
\hline 11/Complete & $\begin{array}{l}\text { RQ: NR } \\
\text { RTS: NR } \\
\text { LQ: NR } \\
\text { LTS: NR }\end{array}$ & $\begin{array}{l}\text { RQ: NR } \\
\text { RTS: RP } \\
\text { LQ: NR } \\
\text { LTS: NR }\end{array}$ & $\begin{array}{l}\text { RQ: NR } \\
\text { RTS: NR } \\
\text { LQ: NR } \\
\text { LTS: NR }\end{array}$ & NA \\
\hline 12/Complete & $\begin{array}{l}\text { RQ: NR } \\
\text { RTS: NR } \\
\text { LQ: RP } \\
\text { LTS: RP }\end{array}$ & $\begin{array}{l}\text { RQ: NR } \\
\text { RTS: NR } \\
\text { LQ: RP } \\
\text { LTS: RP }\end{array}$ & $\begin{array}{l}\text { RQ: NR } \\
\text { RTS: NR } \\
\text { LQ: MLR } \\
\text { LTS: RP }\end{array}$ & $\begin{array}{l}\text { RQ: NR } \\
\text { RTS: NR } \\
\text { LQ: MLR } \\
\text { LTS: RP }\end{array}$ \\
\hline 13/Complete & $\begin{array}{l}\text { RQ: NR } \\
\text { RTS: NR } \\
\text { LQ: RP } \\
\text { LTS: NR }\end{array}$ & $\begin{array}{l}\text { RQ: NR } \\
\text { RTS: NR } \\
\text { LQ: RP } \\
\text { LTS: NR }\end{array}$ & NA & NA \\
\hline 14/Incomplete & $\begin{array}{l}\text { RQ: NR } \\
\text { RTS: NR } \\
\text { LQ: MLR } \\
\text { LTS: MLR }\end{array}$ & $\begin{array}{l}\text { RQ: NR } \\
\text { RTS: NR } \\
\text { LQ: RP } \\
\text { LTS: MLR }\end{array}$ & $\begin{array}{l}\text { RQ: NR } \\
\text { RTS: NR } \\
\text { LQ: RP } \\
\text { LTS: MLR }\end{array}$ & $\begin{array}{l}\text { RQ: NR } \\
\text { RTS: NR } \\
\text { LQ: RP } \\
\text { LTS: MLR }\end{array}$ \\
\hline 15/Incomplete & $\begin{array}{l}\text { RQ: RP } \\
\text { RTS: RP } \\
\text { LQ: RP } \\
\text { LTS: RP }\end{array}$ & $\begin{array}{l}\text { RQ: RP } \\
\text { RTS: NR } \\
\text { LQ: RP } \\
\text { LTS: NR }\end{array}$ & $\begin{array}{l}\text { RQ: RP } \\
\text { RTS: NR } \\
\text { LQ: RP } \\
\text { LTS: RP }\end{array}$ & $\begin{array}{l}\text { RQ: MLR } \\
\text { RTS: NR } \\
\text { LQ: MLR } \\
\text { LTS: MLR }\end{array}$ \\
\hline 16/Complete & $\begin{array}{l}\text { RQ: NR } \\
\text { RTS: RP } \\
\text { LQ: NR } \\
\text { LTS: NR }\end{array}$ & $\begin{array}{l}\text { RQ: NR } \\
\text { RTS: MLR } \\
\text { LQ: RP } \\
\text { LTS: NR }\end{array}$ & $\begin{array}{l}\text { RQ: NR } \\
\text { RTS: MLR } \\
\text { LQ: MLR } \\
\text { LTS: NR }\end{array}$ & NA \\
\hline
\end{tabular}




\begin{tabular}{|c|c|c|c|c|}
\hline 17/Complete & $\begin{array}{l}\text { RQ: NR } \\
\text { RTS: NR } \\
\text { LQ: NR } \\
\text { LTS: NR }\end{array}$ & $\begin{array}{l}\text { RQ: RP } \\
\text { RTS: NR } \\
\text { LQ: NR } \\
\text { LTS: NR }\end{array}$ & NA & NA \\
\hline 18/Complete & $\begin{array}{l}\text { RQ: NR } \\
\text { RTS: RP } \\
\text { LQ: NR } \\
\text { LTS: NR }\end{array}$ & $\begin{array}{l}\text { RQ: NR } \\
\text { RTS: RP } \\
\text { LQ: RP } \\
\text { LTS: NR }\end{array}$ & $\begin{array}{l}\text { RQ: NR } \\
\text { RTS: NR } \\
\text { LQ: NR } \\
\text { LTS: RP }\end{array}$ & $\begin{array}{l}\text { RQ: NR } \\
\text { RTS: NR } \\
\text { LQ: NR } \\
\text { LTS: NR }\end{array}$ \\
\hline
\end{tabular}

MLR: multi-level response; RP: response present; NR: no response; NA: not assessed; Ax: assessment; SCl: spinal cord injury; RQ: right quadriceps; RTS: right triceps surae; LQ: left quadriceps; LTS: left triceps surae

Table 4. Vibration responses from right and left quadriceps and triceps surae during four assessment sessions in 18 participants with $\mathrm{SCl}$

\begin{tabular}{|c|c|c|c|c|}
\hline Participant/Complete vs. Incomplete & First Ax & Second Ax & Third Ax & Fourth Ax \\
\hline 1/Incomplete & $\begin{array}{l}\text { RQ: RP } \\
\text { RTS: RP } \\
\text { LQ: RP } \\
\text { LTS: NR }\end{array}$ & $\begin{array}{l}\text { RQ: RP } \\
\text { RTS: RP } \\
\text { LQ: RP } \\
\text { LTS: RP }\end{array}$ & $\begin{array}{l}\text { RQ: RP } \\
\text { RTS: RP } \\
\text { LQ: RP } \\
\text { LTS: RP }\end{array}$ & $\begin{array}{l}\text { RQ: RP } \\
\text { RTS: NR } \\
\text { LQ: RP } \\
\text { LTS: NR }\end{array}$ \\
\hline 2/Incomplete & $\begin{array}{l}\text { RQ: NR } \\
\text { RTS: NR } \\
\text { LQ: NR } \\
\text { LTS: NR }\end{array}$ & $\begin{array}{l}\text { RQ: MLR } \\
\text { RTS: NR } \\
\text { LQ: NR } \\
\text { LTS: NR }\end{array}$ & NA & NA \\
\hline 3/Incomplete & $\begin{array}{l}\text { RQ: MLR } \\
\text { RTS: NR } \\
\text { LQ: NR } \\
\text { LTS: NR }\end{array}$ & $\begin{array}{l}\text { RQ: MLR } \\
\text { RTS: NR } \\
\text { LQ: MLR } \\
\text { LTS: NR }\end{array}$ & $\begin{array}{l}\text { RQ: MLR } \\
\text { RTS: NR } \\
\text { LQ: MLR } \\
\text { LTS: NR }\end{array}$ & NA \\
\hline 4/Complete & $\begin{array}{l}\text { RQ: NR } \\
\text { RTS: NR } \\
\text { LQ: NR } \\
\text { LTS: NR }\end{array}$ & $\begin{array}{l}\text { RQ: NR } \\
\text { RTS: NR } \\
\text { LQ: NR } \\
\text { LTS: NR }\end{array}$ & $\begin{array}{l}\text { RQ: NR } \\
\text { RTS: NR } \\
\text { LQ: NR } \\
\text { LTS: NR }\end{array}$ & NA \\
\hline 5/Incomplete & $\begin{array}{l}\text { RQ: RP } \\
\text { RTS: NR } \\
\text { LQ: RP } \\
\text { LTS: NR }\end{array}$ & $\begin{array}{l}\text { RQ: NR } \\
\text { RTS: NR } \\
\text { LQ: RP } \\
\text { LTS: NR }\end{array}$ & $\begin{array}{l}\text { RQ: NR } \\
\text { RTS: NR } \\
\text { LQ: NR } \\
\text { LTS: NR }\end{array}$ & $\begin{array}{l}\text { RQ: NR } \\
\text { RTS: NR } \\
\text { LQ: NR } \\
\text { LTS: NR }\end{array}$ \\
\hline $6 /$ Incomplete & $\begin{array}{l}\text { RQ: NR } \\
\text { RTS: NR } \\
\text { LQ: RP } \\
\text { LTS: NR }\end{array}$ & $\begin{array}{l}\text { RQ: RP } \\
\text { RTS: NR } \\
\text { LQ: NR } \\
\text { LTS: NR }\end{array}$ & $\begin{array}{l}\text { RQ: NR } \\
\text { RTS: NR } \\
\text { LQ: NR } \\
\text { LTS: NR }\end{array}$ & $\begin{array}{l}\text { RQ: NR } \\
\text { RTS: NR } \\
\text { LQ: RP } \\
\text { LTS: NR }\end{array}$ \\
\hline 7/Incomplete & $\begin{array}{l}\text { RQ: NR } \\
\text { RTS: NR } \\
\text { LQ: NR } \\
\text { LTS: NR }\end{array}$ & $\begin{array}{l}\text { RQ: NR } \\
\text { RTS: NR } \\
\text { LQ: NR } \\
\text { LTS: NR }\end{array}$ & $\begin{array}{l}\text { RQ: NR } \\
\text { RTS: NR } \\
\text { LQ: NR } \\
\text { LTS: NR }\end{array}$ & NA \\
\hline 8/Complete & $\begin{array}{l}\text { RQ: MLR } \\
\text { RTS: MLR } \\
\text { LQ: MLR } \\
\text { LTS: NR }\end{array}$ & $\begin{array}{l}\text { RQ: MLR } \\
\text { RTS: MLR } \\
\text { LQ: MLR } \\
\text { LTS: MLR }\end{array}$ & $\begin{array}{l}\text { RQ: MLR } \\
\text { RTS: MLR } \\
\text { LQ: MLR } \\
\text { LTS: MLR }\end{array}$ & $\begin{array}{l}\text { RQ: NR } \\
\text { RTS: NR } \\
\text { LQ: NR } \\
\text { LTS: NR }\end{array}$ \\
\hline 9/Complete & $\begin{array}{l}\text { RQ: MLR } \\
\text { RTS: NR } \\
\text { LQ: MLR } \\
\text { LTS: NR }\end{array}$ & $\begin{array}{l}\text { RQ: NR } \\
\text { RTS: NR } \\
\text { LQ: NR } \\
\text { LTS: NR }\end{array}$ & NA & NA \\
\hline 10/Complete & $\begin{array}{l}\text { RQ: NR } \\
\text { RTS: NR } \\
\text { LQ: MLR } \\
\text { LTS: MLR }\end{array}$ & $\begin{array}{l}\text { RQ: NR } \\
\text { RTS: NR } \\
\text { LQ: NR } \\
\text { LTS: MLR }\end{array}$ & NA & NA \\
\hline 11/Complete & $\begin{array}{l}\text { RQ: NR } \\
\text { RTS: NR } \\
\text { LQ: NR } \\
\text { LTS: NR }\end{array}$ & $\begin{array}{l}\text { RQ: NR } \\
\text { RTS: NR } \\
\text { LQ: NR } \\
\text { LTS: NR }\end{array}$ & $\begin{array}{l}\text { RQ: NR } \\
\text { RTS: NR } \\
\text { LQ: NR } \\
\text { LTS: NR }\end{array}$ & NA \\
\hline 12/Complete & $\begin{array}{l}\text { RQ: NR } \\
\text { RTS: RP } \\
\text { LQ: NR } \\
\text { LTS: NR }\end{array}$ & $\begin{array}{l}\text { RQ: RP } \\
\text { RTS: RP } \\
\text { LQ: NR } \\
\text { LTS: NR }\end{array}$ & $\begin{array}{l}\text { RQ: NR } \\
\text { RTS: RP } \\
\text { LQ: NR } \\
\text { LTS: NR }\end{array}$ & $\begin{array}{l}\text { RQ: NR } \\
\text { RTS: NR } \\
\text { LQ: NR } \\
\text { LTS: NR }\end{array}$ \\
\hline 13/Complete & $\begin{array}{l}\text { RQ: NR } \\
\text { RTS: NR } \\
\text { LQ: NR } \\
\text { LTS: NR }\end{array}$ & $\begin{array}{l}\text { RQ: NR } \\
\text { RTS: RP } \\
\text { LQ: NR } \\
\text { LTS: NR }\end{array}$ & NA & NA \\
\hline
\end{tabular}




\begin{tabular}{|c|c|c|c|c|}
\hline 14/Incomplete & $\begin{array}{l}\text { RQ: NR } \\
\text { RTS: NR } \\
\text { LQ: NR } \\
\text { LTS: NR }\end{array}$ & $\begin{array}{l}\text { RQ: RP } \\
\text { RTS: RP } \\
\text { LQ: NR } \\
\text { LTS: RP }\end{array}$ & $\begin{array}{l}\text { RQ: NR } \\
\text { RTS: NR } \\
\text { LQ: NR } \\
\text { LTS: NR }\end{array}$ & $\begin{array}{l}\text { RQ: NR } \\
\text { RTS: NR } \\
\text { LQ: NR } \\
\text { LTS: NR }\end{array}$ \\
\hline $15 /$ Incomplete & $\begin{array}{l}\text { RQ: MLR } \\
\text { RTS: MLR } \\
\text { LQ: MLR } \\
\text { LTS: NR }\end{array}$ & $\begin{array}{l}\text { RQ: NR } \\
\text { RTS: NR } \\
\text { LQ: RP } \\
\text { LTS: NR }\end{array}$ & $\begin{array}{l}\text { RQ: NR } \\
\text { RTS: NR } \\
\text { LQ: RP } \\
\text { LTS: NR }\end{array}$ & $\begin{array}{l}\text { RQ: NR } \\
\text { RTS: NR } \\
\text { LQ: MLR } \\
\text { LTS: NR }\end{array}$ \\
\hline 16/Complete & $\begin{array}{l}\text { RQ: NR } \\
\text { RTS: NR } \\
\text { LQ: NR } \\
\text { LTS: NR }\end{array}$ & $\begin{array}{l}\text { RQ: NR } \\
\text { RTS: RP } \\
\text { LQ: NR } \\
\text { LTS: NR }\end{array}$ & $\begin{array}{l}\text { RQ: NR } \\
\text { RTS: RP } \\
\text { LQ: NR } \\
\text { LTS: NR }\end{array}$ & NA \\
\hline 17/Complete & $\begin{array}{l}\text { RQ: RP } \\
\text { RTS: RP } \\
\text { LQ: MLR } \\
\text { LTS: MLR }\end{array}$ & $\begin{array}{l}\text { RQ: RP } \\
\text { RTS: NR } \\
\text { LQ: MLR } \\
\text { LTS: NR }\end{array}$ & NA & NA \\
\hline 18/Complete & $\begin{array}{l}\text { RQ: MLR } \\
\text { RTS: NR } \\
\text { LQ: MLR } \\
\text { LTS: NR }\end{array}$ & $\begin{array}{l}\text { RQ: NR } \\
\text { RTS: RP } \\
\text { LQ: RP } \\
\text { LTS: NR }\end{array}$ & NA & $\begin{array}{l}\text { RQ: NR } \\
\text { RTS: NR } \\
\text { LQ: NR } \\
\text { LTS: NR }\end{array}$ \\
\hline
\end{tabular}

MLR: multi-level response; NR: no response; RP: response present; NA: not assessed; SCl: spinal cord injury; RQ: right quadriceps; RTS: right triceps surae; LQ: left quadriceps; LTS: left triceps surae

[Figure 3A]. These results show that the ISNCSCI strength assessment only provides information about one element for evaluating treatment efficacy properly in this population. The ISNCSCI "improvement" noted in the current study may be non-specific for indicating clinically useful improvement. Thus, neurophysiological assessments similar to BMCA can increase the resolution of assessment, enabling clinicians to more reliably understand changes in motor control in their patients.

Significant functional recovery after incomplete SCI depends on the plasticity that is occurring through propriospinal network, intraspinal circuits and supraspinal influences through descending systems. Many factors can influence the effectiveness of different rehabilitation strategies in this group of patients, e.g., the level of injury, onset of training and the intensity of the training (how much, how often and how long). To be able to understand which strategy would maximise the activity-dependent plasticity in these patients with significant functional recovery, it would be desirable to undertake routine neurophysiological assessment to collect valuable information from this population during their rehabilitation period. The results reported here illustrate the variability of responses between patients and highlight the importance of collection of larger datasets for interpretation of changes over time and in response to different rehabilitation strategies.

In this study, all 10 participants who were categorised as having complete SCI showed some sub-clinical supraspinal influences over the muscles below the level of injury. This is in line with previous studies ${ }^{[25]}$. It has been shown that a TVR response in people with clinically complete SCI can be considered as a subclinical supraspinal response so they should be classified as having discomplete $\mathrm{SCI}^{[2,26,27]}$. In the present study, Participants 8, 10, 12, 13, 16, 17 and 18 showed TVR in 1-4 targeted muscles, which indicates that they should be categorised as discomplete. Gillies et al. ${ }^{[28]}$ showed that the TVR could be observed in a cat with SCI only if the lateral vestibulospinal and pontine reticulospinal tracts were intact. It has been argued that, during vibration, the sensory information is transmitted to the brainstem, the reticular formation and associated tracts, as well as other parts of the brain that all are involved in controlling this response ${ }^{[27]}$. In the present study, Participants 8, 10, 12, 13, 16, 17 and 18 showed TVR in 1-4 targeted muscles. These subclinical responses should not be ignored as they might open a new window for exploring new rehabilitation techniques to improve the supraspinal influences over the muscles under the level of injury that these patients could benefit ${ }^{[3,29]}$. 
Another marker for the existence of supraspinal influences is the tendon tap response ${ }^{[25,30]}$. In this study, Participants 4, 8, 10,11,12,13,16,17 and 18 showed tendon tap responses in at least one of the assessed muscles without extending to other spinal segments. This response has been reported in previous studies as well $^{[14,31]}$. There is significant supraspinal influence on inhibitory interneurons at different spinal segments and propriospinal neurons that can extend to other segmental levels, as well as a direct influence on alpha and gamma motoneurons. It has been shown that a reduction of supraspinal influences over propriospinal interneuron networks increases their excitability, which in turn increases the possibility of motor unit activation in other spinal levels including on the contralateral side $\mathrm{s}^{[32]}$.

In the present study, participants in the whole-body training group completed 12 weeks of training including trunk, upper and lower limb exercises and LT, FES-assisted cycling. These participants received FES, which increased the sensory inputs to the propriospinal network and intraspinal circuits through dromic and anti-dromic currents in the stimulated nerves and sensory feedback from the contracted muscles and joint receptors post-muscle contractions ${ }^{[33]}$. Plasticity of these networks plays a significant role in functional recovery in patients with incomplete SCI by forming new connections and re-establishing corticospinal connections to the affected muscles ${ }^{[34]}$. The increased sensory inputs to the spinal cord could increase the excitability of the propriospinal network and promote multi-level muscle co-activations or reflex responses, which can adversely decrease the SIs for different tasks. However, it is unlikely that this was the case in the present study, as these responses were seen in participants in both groups. As we assessed a small number of patients, this speculation needs to be confirmed in larger studies.

\section{Limitations of the study}

BMCA requires specialised equipment and expertise in collecting and analysing the data, which may not be readily available at all sites. The sample size in this study was low as the BMCA assessments were limited to participants at only one site of a multi-centre trial. The number of tasks was limited to just four unilateral tasks. In future studies, other lower limb movements should also be assessed, e.g., hip abduction/adduction. In addition, all the tasks were completed in the supine position in order to standardise the testing position. This could affect the control of anti-gravity movements, e.g., hip and knee flexion, and increase the intersubject variability significantly. Other factors were the variability in time post-injury within this group of participants (1.5-50 years), and their unique patterns of injury, which may also affect the interpretation of the data.

In conclusion, knowledge about how to improve function in people with SCI is growing, with new therapeutic approaches, modification of previous approaches and new technologies to facilitate compensatory function. In line with this, the need for objective evaluation of the effectiveness of these therapeutic approaches will also grow. Neurophysiological assessment will assist clinicians to monitor their patients' progress during rehabilitation programs with more resolution and potentially lead to individualised adjustment to optimise rehabilitation outcomes. BMCA is a valuable objective assessment tool that can refine the clinical evaluation of patients with SCI and assist in maximising their functional capabilities. Reporting the BMCA findings after different therapeutic techniques and rehabilitation programs, even in a small number of patients, will help to increase our knowledge of the effects of those interventions on movement patterns and residual supraspinal effects.

\section{DECLARATIONS}

\section{Authors' contributions}

Designed the study, obtained funding, collected and interpreted the data, and revised the manuscript: Galea M

Collected, analysed and interpreted data, prepared the manuscript and all tables and figures, and revised the manuscript: Zoghi M 


\section{Availability of data and materials}

Data can be made available on application to the authors.

\section{Financial support and sponsorship}

The study was funded by the Transport Accident Commission (Victorian Neurotrauma Initiative), and the University of Melbourne.

\section{Conflicts of interest}

All authors declared that there are no conflicts of interest.

\section{Ethical approval and consent to participate}

The study was approved by the Human Research Ethics Commitees of Austin Health and the University of Melbourne.

\section{Consent for publication}

Not applicable.

\section{Copyright}

(c) The Author(s) 2019.

\section{REFERENCES}

1. Behrman AL, Harkema SD. Physical rehabilitation as an agent for recovery following spinal cord injury. Phys Med Rehabil Clin N Am 2007;18:183-202.

2. Sherwood AM, Dimitrijevic MR, McKay WB. Evidence of subclinical brain influence in clinically complete spinal cord injury: discomplete SCI. J Neurol Sci 1992;110:90-8.

3. Gill ML, Grahn PJ, Calvert JS, Linde MB, Lavrov IA, et al. Neuromodulation of lumbosacral spinal networks enables independent stepping after complete paraplegia. Nat Med 2018;24:1677-82.

4. Kakulas BA. Pathology of spinal injuries. Cent Nerv Syst Trauma 1984;1:117-29.

5. Dimitrijevic MR. Residual motor functions in spinal cord injury. Adv Neurol 1988;47:138-55.

6. Mayr W, Krenn M, Dimitrijevic MR. Motor control of human spinal cord disconnected from the brain and under external movement. Adv Exp Med Biol 2016;957:159-71.

7. Taccola G, Sayenko D, Gad P, Gerasimenko Y, Edgerton VR. And yet it moves: recovery of volitional control after spinal cord injury. Prog Neurobiol 2018;160:64-81.

8. Minassian K, Hofstoetter US. Spinal cord stimulation and augmentative control strategies for leg movement after spinal paralysis in humans. CNS Neurosci Ther 2016;22:262-70.

9. Harkema SJ. Neural plasticity after human spinal cord injury: application of locomotor training to the rehabilitation of walking. Neuroscientist 2001;7:455-68.

10. Behrman AL, Harkema SD. Physical rehabilitation as an agent for recovery following spinal cord injury. Phys Med Rehabil Clin N Am 2007; 18:183-202.

11. McDonald JW, Becker D, Sadowsky CL, Jane JA Sr, Conturo TE, et al. Late recovery following spinal cord injury. Case report and review of the literature. J Neurosurg 2002;97:252-65.

12. Dimitrijevic MR, Dimitrijevic MM, Faganel J, Sherwood AM. Suprasegmentally induced motor unit activity in paralyzed muscles of patients with established spinal cord injury. Ann Neurol 1984;16:216-21.

13. Sherwood AM, McKay WB, Dimitrijevic MR. Motor control after spinal cord injury: assessment using surface EMG. Muscle Nerve 1996;19:966-79.

14. Sherwood AM, Dimitrijevic MR, Bacia T, McKay WB. Characteristics of the vibratory reflex in humans with reduced suprasegmental influence due to spinal cord injury. Restor Neurol Neurosci 1993;5:119-29.

15. Cioni B, Dimitrijevic MR, McKay WB, Sherwood AM. Voluntary supraspinal suppression of spinal reflex activity in paralyzed muscles of spinal cord injury patients. Exp Neurol 1986;93:574-83.

16. Kakulas A. The applied neurobiology of human spinal cord injury: a review. Paraplegia 1988;26:371-9.

17. Galea MP, Dunlop SA, Geraghty T, Davis GM, Nunn A, et al. SCIPA full-on: a randomized controlled trial comparing intensive whole-body exercise and upper body exercise after spinal cord injury. Neurorehab Neural Re 2018;32:557-67.

18. Kirshblum SC, Burns SP, Biering-Sorensen F, Donovan W, Graves DE, et al. International standards for neurological classification of spinal cord injury (revised 2011). J Spinal Cord Med 2011;34:535-46.

19. Betz R, Biering-Sørensen F, Burns SP, Donovan W, Graves DE, et al. The 2019 revision of the International Standards for Neurological Classification of Spinal Cord Injury (ISNCSCI) - What's new? Spinal Cord 2019;57:815-7.

20. Lee DC, Lim HK, McKay WB, Priebe MM, Holmes SA, et al. Toward an objective interpretation of surface EMG patterns: a 
voluntary response index (VRI). J Electromyogr Kinesiol 2004;14:379-88.

21. Zoghi M, Galea M, Morgan D. A Brain Motor Control Assessment (BMCA) protocol for upper limb function. PLoS One 2013;8:e79483.

22. Galea MP, Dunlop SA, Davis GM, Nunn A, Geraghty T, et al. Intensive exercise program after spinal cord injury ("Full-On”): study protocol for a randomized controlled trial. Trials 2013;14:291.

23. Black J, Baharestani M, Cuddigan J, Dorner B, Edsberg L, et al. National Pressure Ulcer Advisory Panel's updated pressure ulcer staging system. Dermatol Nurs 2007;19:343-9.

24. Harkema S, Behrman A, Barbeau H, editors. Locomotor training: principles and practice. New York: Oxford University Press; 2011.

25. McKay WB, Lim HK, Priebe MM, Stokic DS, Sherwood AM. Clinical neurophysiological assessment of residual motor control in post-spinal cord injury paralysis. Neurorehabil Neural Repair 2004;18:144-53.

26. Dimitrijevic MR. Neurophysiology in spinal cord injury. Paraplegia 1987;25:205-8.

27. Dimitrijevic MR, Spencer WA, Trontelj JV, Dimitrijevic M. Reflex effects of vibration in patients with spinal cord lesions. Neurology 1977;27:1078-86

28. Gillies JD, Burke DJ, Lance JW. Tonic vibration reflex in the cat. J Neurophysiol 1971;34:252-62.

29. Cote MP, Murray M, Lemay MA. Rehabilitation strategies after spinal cord injury: inquiry into the mechanisms of success and failure. J Neurotrauma 2017;34:1841-57.

30. Lundberg A. Multisensory control of spinal reflex pathways. Prog Brain Res 1979;50:11-28.

31. Dimitrijević MR, Nathan PW. Studies of Spasticity in Man. 1. Some features of spasticity. Brain 1967;90:1-30.

32. Kern H, McKay WB, Dimitrijevic MM, Dimitrijevic MR. Motor control in the human spinal cord and the repair of cord function. Curr Pharm Des 2005;11:1429-39.

33. Dobkin BH. Do electrically stimulated sensory inputs and movements lead to long-term plasticity and rehabilitation gains? Curr Opin Neurol 2003;16:685-91.

34. Bareyre FM, Kerschensteiner M, Raineteau O, Mettenleiter TC, Weinmann O, et al. The injured spinal cord spontaneously forms a new intraspinal circuit in adult rats. Nat Neurosci 2004;7:269-77. 